\title{
Exogenous surfactant enhances mucociliary clearance in the anaesthetized dog
}

\author{
G.T. De Sanctis*, R.P. Tomkiewicz**, B.K. Rubin , S. Schürch ${ }^{\dagger}$, M. King**
} Exogenous surfactant enhances mucociliary clearance in the anaesthetized dog. G.T. De
Sanctis, R.P. Tomkiewicz, B.K. Rubin, S. Schürch, M. King. CERS Journals Ltd 1994.
ABSTRACT: Therapy with exogenous surfactants is currently used for the treatment
of respiratory distress syndrome of the newborn (RDS) and is under investigation
for treatments related to adult RDS. However, the possible use of exogenous
surfactant as a means of enhancing mucus clearance in other respiratory diseases
has not been addressed. We therefore studied the effects of an artificial surfactant
(Curosurf) on in vivo tracheal mucus velocity in intubated pentobarbital-anaesthetized
dogs.

Five dogs were randomly administered, on separate occasions, either vehicle (saline) or $10 \mathrm{mg}$ Curosurf by means of local instillation via a catheter into the right lung. Tracheal mucus was collected by inserting a soft-bristled cytology brush to the level of the carina, and analysed for viscoelasticity by microrheometry. Mucociliary

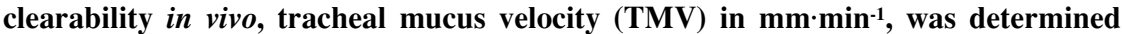
by bronchoscopic observation of charcoal marker particle transit times. The initial placement of charcoal was at the same level of the lower trachea that mucus was collected from. The effect of ciliary beat frequency was assessed on the frog palate assay by a videoscopic technique.

In the dog, TMV was significantly increased after administration of surfactant. The values of TMV in the vehicle- and surfactant-treated dogs were $6.3 \pm 4.0 \mathrm{vs}$ $25.6 \pm 6.5 \mathrm{~mm} \cdot \mathrm{min}^{-1}(\mathrm{SD})$, respectively. There were no discernible differences between prevehicle and postvehicle TMV values, and no significant differences in any mucus viscoelastic parameter, as determined by magnetic rheometry. There were also no significant changes in the viscoelastic or surface properties of mucus incubated with surfactant in vitro; however, ciliary beat frequency increased following administration of surfactant in the frog palate assay (from 3.6 \pm 0.4 to $8.5 \pm 3.2 \mathrm{~Hz}$ ). The stimulation of TMV may be due either to a direct effect on ciliary function, to a reduction in the viscosity of the periciliary fluid, or to a more efficient interaction between the mucus and the cilia.

In view of these results, we suggest that exogenous surfactant administration may provide a new therapeutic approach in treating conditions of impaired mucus clearance.

Eur Respir J., 1994, 7, 1616-1621.
*Brigham \& Women's Hospital and Harvard Medical School, Boston, USA. **Pulmonary \& Cell Biology Research Group, University of Alberta, Edmonton, Canada. 'St. Louis University Pediatric Research Institute and Cardinal Glennon Children's Hospital, St. Louis, USA. Respiratory Research Group, University of Calgary, Calgary, Canada.

Correspondence: M. King

173 Heritage Medical Research Center University of Alberta

Edmonton

Canada T6G 2S2

Keywords: Ciliary beat frequency mucociliary clearance mucus viscoelasticity pulmonary surfactant

Received: May 251993

Accepted after revision June 61994

This work was supported by the MRC of Canada, the Canadian Cystic Fibrosis Foundation, and the Cardinal Glennon Hospital Pediatric Pulmonary Research Fund. G.T. De Sanctis is a recipient of a MRC of Canada Fellowship. R.P. Tomkiewicz is a recipient of a Canadian Cystic Fibrosis Foundation Fellowship.
The airways of the tracheobronchial tree are largely covered by a thin blanket of mucus. Mucus is a viscoelastic secretion that protects the underlying mucosa from dehydration, whilst trapping inhaled particles that come into contact with it. The mucociliary clearance mechanism is primarily responsible for the removal of mucus from the airways. Our understanding of the mechanism of this clearance is based on the two-fluid model of mucus [1-3]. The upper layer of mucus is assumed to be a viscoelastic gel, consisting principally of cross-linked glycoproteins. The lower serous layer, which bathes the cilia, is a sol phase. The mucus flows on the sol layer and is transported by the cilia, which beat in a synchronized fashion.

The rate at which mucus is cleared is dependent on various factors, including the viscoelasticity of the mucus $[3,4]$. Several studies have suggested that the presence of phospholipids in the airways may modify the clearance of mucus [5-10]. The existence of surfactant films has been demonstrated in the airways of several species by electron microscopy $[10,11]$, and in horses and sheep by in situ surface tension measurements [10, 12]. Furthermore, both nonciliated bronchiolar cells [13], and bronchial mucous glands [14], have been shown to produce surface active materials. Although the functional significance of surfactant on mucociliary transport in vivo has not been studied, when surfactant obtained from porcine lung was sprayed on excised frog palate, there was a significant increase in transport rate when compared to saline [5].

Surfactant therapy has been widely accepted as being valuable in the treatment of neonatal respiratory distress syndrome (RDS) $[15,16]$, and is under active investigation for the treatment of adult RDS. Currently, lung 
surfactant has not been used for the treatment of impaired mucociliary clearance. The present study reports experiments designed to test the hypothesis that airway surfactant influences mucociliary transport in an in vivo model, and to determine whether any enhancement can be attributed to alterations in mucus viscoelasticity or to increased ciliary activity.

\section{Methods}

\section{Study design}

Five beagle dogs, weight $10-15 \mathrm{~kg}$, were studied on two occasions each. The dogs were anaesthetized with sodium pentobarbital $\left(25 \mathrm{mg} \cdot \mathrm{kg}^{-1}\right.$ i.v., supplemented as required), placed supine and intubated with a shortened No. 9 endotracheal tube (ETT). Each dog was randomly administered either vehicle (normal saline - $0.15 \mathrm{M} \mathrm{NaCl}$ ) or $10 \mathrm{mg}$ of a surfactant preparation $\left(\right.$ Curosurf $^{\circledR}$, a gift from T. Curstedt of the Karolinska Institute, Stockholm, Sweden) suspended in $0.15 \mathrm{M} \mathrm{NaCl}$. Topical instillation of $5 \mathrm{ml}$ of $2 \mathrm{mg} \cdot \mathrm{ml}^{-1}$ solution was carried out over a 2 min period of time via a catheter into the right mainstem bronchus, approximately $1 \mathrm{~cm}$ below the level of the carina. The catheter was equipped with small side-ports, ensuring a uniform distribution of surfactant over the entire circumference of the airway. Tracheal mucus linear velocity measurements and tracheal mucus collection were performed prior to and after administration of either vehicle or surfactant.

Curosurf is a preparation from porcine lung containing alveolar phospholipids and apoproteins, isolated by liquid-gel chromatography [17]. It contains about $99 \%$ polar lipids (25\% dipalmitoylphosphatidylcholine) and $1 \%$ hydrophobic proteins. The surfactant associated proteins SP-B and SP-C are present in approximate molar ratio $1: 2 ; \mathrm{SP}-\mathrm{A}$ is absent.

\section{Procedures and techniques}

Tracheal mucus linear velocity (TMV). Direct evaluation of mucociliary clearability in vivo, TMV in $\mathrm{mm} \cdot \mathrm{min}^{-1}$, was determined by bronchoscopic observation of charcoal marker particle (Sigma No. C 5260) transit times (leading edge) in the lower trachea [18]. The initial placement of charcoal was on the right side of the posterior membrane of the lower trachea, $1 \mathrm{~cm}$ above the carina, i.e. approximately $2 \mathrm{~cm}$ downstream from the site of surfactant deposition. TMV measurements were initiated 10 min after surfactant administration; transit times were generally $10 \mathrm{~min}$ or less, depending on the rapidity with which the charcoal front approached the inlet of the endotracheal tube.

Mucus collection. Mucus was collected from the same level of the lower trachea, from the left side after baseline TMV measurement and from the right side following Curosurf or placebo TMV measurements. A modified cytology brush technique was used, by placing a cytology brush (No. 151 Mill Rose, Mentor, OH, USA), guided by a bronchoscope against the wall of the airway, and removing the brush once it was covered with sufficient mucus for analysis [18]. The sampling time was normally $10 \mathrm{~min}$. Mucus collections and TMV were carried out at comparable levels, but on opposite sides to avoid interference. For each experiment, the mucus samples were frozen in paraffin oil at $-80^{\circ} \mathrm{C}$ until analysed for bulk viscoelasticity by magnetic rheometry. The effect of additional surfactant on untreated canine tracheal mucus in vitro was further assessed by layering 3 $\mu \mathrm{l}$ of Curosurf solution $\left(1 \mathrm{mg} \cdot \mathrm{ml}^{-1}\right)$, or saline vehicle, onto $30 \mu \mathrm{l}$ of mucus ( $\mathrm{n}=7$ sample pairs) and incubating this mixture at $35^{\circ} \mathrm{C}$ for $10 \mathrm{~min}$ without stirring, before measuring the viscoelastic and surface properties of the secretions as detailed below.

Mucus dynamic viscoelasticity. The magnetic microrheometer technique was employed to measure the bulk viscosity and elasticity of microlitre quantities of mucus [19]. A $100 \mu \mathrm{m}$ steel ball was carefully positioned in a $5 \mu \mathrm{l}$ sample of mucus, and the motion of this sphere under the influence of an electromagnet was used to determine the rheological properties of the mucus. For that purpose, the image of the steel ball was magnified and projected onto a pair of photocells, the output of which was amplified and transmitted to an oscilloscope. By plotting the displacement of the ball (strain) against the magnetic driving force (stress), the dynamic viscoelastic properties of the mucus were determined. These included mechanical impedance or mucus rigidity $\left(\mathrm{G}^{*}\right)$, reported here in log scale, expressing the vector sum of viscosity and elasticity, and loss tangent $(\tan \delta)$, reflecting the ratio of viscosity to elasticity. The loss tangent is inversely related to mucus recoil $[4,19]$. Both viscoelastic parameters were evaluated at low $\left(1 \mathrm{rad} \cdot \mathrm{s}^{-1}\right)$ and high $\left(100 \mathrm{rad} \cdot \mathrm{s}^{-1}\right)$ oscillation frequency.

Mucus dynamic adhesivity (surface mechanical impedance). The rolling ball technique is used in industry to measure the adhesiveness of glue strips. The magnetic microrheometer and computer measurement software was modified in order to make rolling ball adhesion measurements on small specimens of mucus. The $100 \mu \mathrm{m}$ steel sphere was placed on the surface of the mucous layer without using a paraffin oil cover. The magnetic force needed to achieve steady-state oscillation of the sphere across the surface of the sample was used to calculate the surface mechanical impedance $\left(\mathrm{Gs}^{*}\right)$ at 1 and $10 \mathrm{rad} \cdot \mathrm{s}^{-1}$.

Surface wettability by mucus. The wettability of a hydrophobic surface (TFE; Teflon (1)) and a hydrophilic surface (acid washed glass slide) was determined by placing droplets of mucus on these surfaces (sessile drop technique). A $15 \mu$ l sample of mucus was placed on either the TFE strip or the glass slide and positioned in the field of a microscope equipped with a videocamera and a video processing system. Output from these images was recorded and processed through an image analyser to determine the contact angle $(\theta)[7,9]$. 
Table 1. - Tracheal mucus rheology with in vivo Curosurf administration (10 mg) $(\mathrm{n}=5)$

\begin{tabular}{|c|c|c|c|c|}
\hline \multirow[b]{2}{*}{ Parameter } & \multicolumn{2}{|c|}{ Saline } & \multicolumn{2}{|c|}{ Curosurf } \\
\hline & Pre & Post & Pre & Post \\
\hline $\log \mathrm{G}^{*} 1 \mathrm{rad} \cdot \mathrm{s}^{-1}$ & $2.16 \pm 0.31$ & $2.51 \pm 0.14$ & $2.40 \pm 0.47$ & $2.16 \pm 0.36$ \\
\hline $\tan \delta \mathrm{rad} \cdot \mathrm{s}^{-1}$ & $0.28 \pm 0.04$ & $0.28 \pm 0.03$ & $0.25 \pm 0.05$ & $0.26 \pm 0.04$ \\
\hline $\log \mathrm{G}^{*} 100 \mathrm{rad} \cdot \mathrm{s}^{-1}$ & $2.65 \pm 0.24$ & $3.02 \pm 0.15$ & $2.90 \pm 0.42$ & $2.73 \pm 0.36$ \\
\hline $\tan \delta 100 \mathrm{rad} \cdot \mathrm{s}^{-1}$ & $1.25 \pm 0.16$ & $1.26 \pm 0.17$ & $1.10 \pm 0.34$ & $1.23 \pm 0.13$ \\
\hline
\end{tabular}

All values are presented as mean \pm SD. $G^{*}$ : mucus rigidity, reported in log scale, expressing the vector sum of viscosity and elasticity; $\tan \delta$ : loss tangent, reflecting ratio of viscosity to elasti-city.

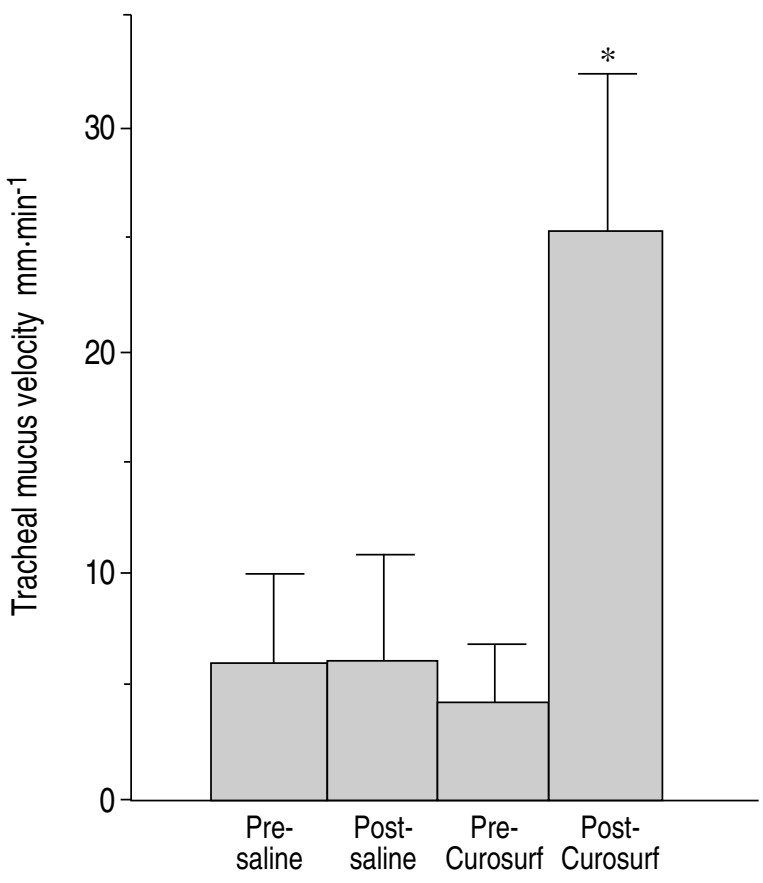

Fig. 1. - Tracheal mucus velocity (TMV) in five dogs before and after treatment with either saline or Curosurf (mean $\pm \mathrm{SD})$. The increase in TMV with Curosurf was significant $(\mathrm{p}<0.05$ with respect to preCurosurf, as well as with respect to post-saline).

Ciliary beat frequency $(C B F)$ on frog palate. The same concentration of Curosurf as administered to the dogs $(2$ $\mathrm{mg} \cdot \mathrm{ml}^{-1}$ ) was applied directly to excised frog palate epithelia $(n=6)$. The total volume delivered was adjusted to reflect the ratio of the surface area of the frog palate to the estimated surface area of the trachea and major bronchi of the dog [20], i.e. 4 vs $300 \mathrm{~cm}^{2}$, respectively. Ciliary beating was viewed microscopically and recorded on videotape for analysis by a photometric method [21].

Statistical methods. Statistical analysis was performed using the StatView II statistics package (Abacus Concepts, Berkeley, CA, USA) and a Macintosh II computer (Apple Computer, Cupertino, CA, USA). All results are presented as mean \pm standard deviation (SD). Comparisons between baseline, saline and surfactant values were assessed using analysis of variance (ANOVA) followed by paired t-tests as appropriate. In all cases $\mathrm{p}<0.05$ was considered significant.

\section{Results}

Samples of mucus collected before and after administration of Curosurf or vehicle were analysed for their rheological and transport properties (table 1). A comparison of the change in tracheal mucus velocity (TMV) prior to and following each treatment revealed a significant treatment effect $(p<0.05)$, whereas the effect of vehicle treatment was nonsignificant (fig. 1). There were no significant differences in TMV between baseline measurements taken prior to administration of either saline or surfactant. As illustrated in figure 1, there was approximately a 4-6 fold increase (compared to vehicle or baseline values, respectively) in TMV in dogs treated with surfactant (25.6 vs $4.4 \mathrm{~mm} \cdot \mathrm{min}^{-1}$ ).

Baseline ciliary beat frequency $(\mathrm{CBF})$ on the extirpated frog palate at room temperature was $3.6 \pm 0.4 \mathrm{~Hz}$. Surfactant treatment resulted in an increase in CBF up to $8.5 \pm 3.2 \mathrm{~Hz}(\mathrm{p}<0.01)$, whereas the $\mathrm{CBF}$ after vehicle control did not significantly differ from the baseline value; $3.2 \pm 0.1$ vs $4.0 \pm 0.6 \mathrm{~Hz}$.

Whilst there were significant treatment-induced effects in TMV, there were no comparable effects on any of the rheological parameters evaluated in this study. A comparison of $\log \mathrm{G}^{*}$ (a measure of rigidity) at both 1 and 100 $\mathrm{rad} \cdot \mathrm{s}^{-1}$ after treatment with either vehicle or surfactant (table 1) revealed a tendency towards less rigid mucus in the surfactant-treated group (log $\mathrm{G}^{*} 1$ : postsurfactant vs postsaline, $\mathrm{p}=0.055 ; \log \mathrm{G}^{*} 100$ : postsurfactant $v s$ postsaline, $\mathrm{p}=0.053$ ). However, there were no significant changes observed in mucus bulk viscoelasticity or surface mechanical impedance after in vitro incubation with surfactant or saline vehicle (table 2). The changes in contact angle $\left(\theta^{\circ}\right)$ with hydrophilic and hydrophobic surfaces following surfactant treatment were nonsignificant.

Table 2. - Tracheal mucus properties with in vitro Curosurf administration $\left(0.1 \mathrm{mg} \cdot \mathrm{ml}^{-1}\right.$ final concentration) $(\mathrm{n}=7)$

\begin{tabular}{lll}
\hline Parameter & Saline & Curosurf \\
\hline $\log \mathrm{G}^{*} 1 \mathrm{rad} \cdot \mathrm{s}^{-1}$ & $2.57 \pm 0.25$ & $2.70 \pm 0.35$ \\
$\tan \delta 1 \mathrm{rad} \cdot \mathrm{s}^{-1}$ & $0.37 \pm 0.17$ & $0.33 \pm 0.08$ \\
$\log \mathrm{Gs} * 1 \mathrm{rad} \cdot \mathrm{s}^{-1}$ & $2.57 \pm 0.43$ & $2.82 \pm 0.38$ \\
$\theta^{\circ}-\mathrm{TFE}$ & $97.0 \pm 12.2$ & $95.9 \pm 7.5$ \\
$\theta^{\circ}-$ glass & $42.7 \pm 7.3$ & $41.9 \pm 10.9$ \\
\hline
\end{tabular}

All values are presented as mean \pm SD. $\theta$ : contact angle, surface wettability by mucus; Gs*: surface mechanical impedance. TFE: Teflon hydrophobic surface; glass: hydrophilic surface. For further abbreviations see legend to table 1. 


\section{Discussion}

These studies are the first to examine the influence of surfactant treatment on mucociliary clearance in vivo. In vitro studies have suggested that surfactants may enhance mucociliary clearance $[5,6,8,22,23]$. The present study evaluates the contribution of surfactant treatment in vivo on mucociliary clearance and mucus rheology in anaesthetized dogs. The essential findings that we report here are a substantial enhancement of the clearance rate of tracheal mucus with surfactant treatment, associated with increased ciliary beat frequency in the frog palate, but without significant changes in the bulk viscoelastic properties of the mucus.

MorgenRoth and Bolz [6] examined the airway coating of human bronchial tissue, and demonstrated the presence of phospholipid membranes in the sol phase. The authors speculated that the surfactant was secreted via the bronchial system and probably served to stabilize the sol phase, whilst influencing the sliding capacity of the gel phase. In a recent study using electron microscopy, MERCER et al. [11] demonstrated the existence of a surfactant lining layer at the mucus-air interface in bronchioles of the rat and in smaller bronchi of human airways. Other studies have demonstrated the presence of a surfactant film at the mucus-air interface in the airways of sheep and horse by in situ surface tension measurements [10,12], and reticulated surfactant structures have been demonstrated in the periciliary fluid above epithelial cells [24].

Using electron microscopy, GeHR et al. [10] have shown the existence of a bilayer separating the periciliary fluid (sol phase) and the mucus (gel phase) at locations where both phases were preserved. Transmission electron micrographs demonstrated that particles were displaced from the air-mucus interface into the aqueous subphase. Morphological and surface tension data suggested that the surfactant films provided the interfacial driving force for the displacement of the particles. The authors concluded that the mechanism responsible for clearing these retained particles resided with the airway macrophages, which take up the particles and clear them slowly out of the lung. This slow mechanism, however, cannot be related to the rapid increase in tracheal mucus velocity observed in the dogs in the present experiment after treatment with surfactant.

It has also been shown in vitro that compression and expansion of surfactant films in a Langmuir trough causes net movement of particles, thereby supporting the hypothesis that surfactant may enhance particle clearance in the lung [22]. However, this mechanism is based on the existence of a changing surface tension-area relationship, which exists only in the alveoli and, probably, in peripheral airspaces but not in the trachea and major bronchi. Thus, the condition for particle transport by periodically changing surface tension does not apply in the central airways and trachea [21].

Several other studies have proposed an entirely different mechanism of action to explain the effects of surfactant on mucociliary transport [5, 6, 9]. Allegra et al. [5] reported a significant increase in in vitro mucociliary transport on frog palates sprayed with surfactant. Mucociliary transport was not evaluated under control conditions; i.e. postsurfactant to baseline. The increase in mucociliary rate in this study has been ascribed to the "anti-adhesive" effects of surfactant. Thus, surfactant may act to facilitate sliding of the gel phase on the periciliary fluid. Alternatively, a surfactant layer between the sol and mucous gel phases could facilitate the efficient transfer of energy from the beating cilia to the mucus by decreasing the frictional loss of ciliary kinetic energy and preventing entanglement of the cilia with the adhesive mucous gel.

The surface tension of the surface active film due to phospholipids is found to be much lower than that of other biopolymers. For example, HoLm et al. [25] have determined the plateau surface tension for various protein solutions (e.g. albumin 49, haemoglobin 42 and fibrinogen $\left.45 \mathrm{mN} \cdot \mathrm{m}^{-1}\right)$. These values are much higher than the $25-30 \mathrm{mN} \cdot \mathrm{m}^{-1}$ obtained in vitro for $0.4-1.0 \mathrm{mg} \cdot \mathrm{ml}^{-1}$ Curosurf solution at $37^{\circ} \mathrm{C}$ [26]. In horse trachea in vivo [27], at concentrations and volume of Curosurf equivalent to those used in the dog, a surface tension of 23-25 $\mathrm{mN} \cdot \mathrm{m}^{-1}$ is obtained.

In model systems [28], nonuniformity in surfactant concentration gives rise to gradients in surface tension, which results in a net force acting to pull the surface layer in the direction of higher surface tension. This motion is resisted by a viscous shear stress acting in the liquid layer just beneath the interface. This shear stress, in turn, drags the liquid within the entire liquid layer in the direction of higher surface tension, producing a bulk convection of liquid and surfactant (Marangoni effect). The concentration of Curosurf in the airways is not known, but assuming the $5 \mathrm{ml}$ bolus of surfactant delivered to the bronchus spreads completely over the tracheobronchial surface (estimated $300 \mathrm{~cm}^{2}$ in the dog) [20], then the surface concentration of Curosurf that could be achieved is $c a .300 \mathrm{mg} \cdot \mathrm{m}^{-2}$.

Recent experiments with the captive bubble technique [29] have shown that film material for 3-5 monolayers is associated with the surface active film after adsorption to equilibrium ( $\left.c a .25 \mathrm{mN} \cdot \mathrm{m}^{-1}\right)$. Since the surface tension in the trachea $[12,27]$ is close to this value, we may assume a film thickness of 3-5 monolayers at the airwall interface, i.e. $12-20 \mathrm{~nm}$, taking the length of a phospholipid molecule to be about $4 \mathrm{~nm}$. This thickness of phospholipid film translates into $12-20 \mathrm{mg} \cdot \mathrm{m}^{-2}$. Thus, the amount of exogenous phospholipid administered was more than sufficient to coat the entire tracheobronchial tree of the dog.

Because Curosurf stimulated the ciliary beat frequency when applied to isolated frog palate, we speculate that this may, partially, explain the significant increase in tracheal mucus velocity in the dog. Specifically, if the enhancement of TMV is due to ciliary factors, it could result from a direct effect on ciliary function [30], or to a reduction in the viscosity of the sol phase (for which there is no evidence as yet), or to a change in the strength of the interaction (adhesiveness) between the mucus and the cilia, as recently suggested $[9,31]$.

Alternatively, it has been proposed that airway lipids 
may function to alter the viscoelastic properties of mucus, making it more fluid [32, 33]. A recent study examining mucus rheology and transport in neonatal respiratory distress syndrome and the effect of surfactant therapy supported the above hypothesis [8]. Surfactant treatment in neonates with respiratory distress syndrome appeared to improve mucus clearability, as assessed in vitro, when compared to untreated neonates, mainly due to a treatment-induced effect on mucus rigidity. Alterations in sputum phospholipid content have been correlated with changes in rheological properties of airway secretions in cystic fibrosis (CF) [33]; however, this does not necessarily indicate that adding phospholipids to $\mathrm{CF}$ sputum would correct the viscosity defect. In our own experiments, we observed that the addition of Curosurf to dog tracheal mucus did not significantly alter the bulk viscoelastic properties. The modest effect on mucus viscoelasticity in samples collected in vivo, amounting to about a twofold decrease in mucus rigidity, would be associated with no more than about a $50 \%$ increase in TMV according to model studies [3, 4], far from the 4-6 fold increase that was observed. It may also be that no significant effects on mucus viscoelasticity or surface properties were observed after the in vitro addition of surfactant, partly because the storage of mucus samples in light paraffin oil could, of itself, change at least the surface properties, and could partially obscure the effect of adding exogenous surfactant to mucus that had been stored in this manner. It is also possible that, in some way, the topical application of surfactant to the airway epithelial surface could cause the release of a more watery mucus, similar to that noted after low dose cholinergic [4] or peptidergic [34] stimulation.

It may be surprising that a 4-6 fold increase in TMV could have been induced in a normal mucociliary system. However, general anaesthesia in dogs is associated with suppression of mucociliary clearance [35], and in humans this occurs without significant changes in mucus viscoelasticity [36]. In normal dogs, or even in healthy normal humans, considerable stimulation of mucociliary clearance rate is possible [20,37].

It is only in the last few years that the surface properties of mucus have been recognized as separate and distinct from bulk viscoelasticity. The surface properties are believed to be of critical importance for most aspects of mucus function - its clearance by airflow and ciliary mechanisms, as well as its cytoprotective function [7, 9, 31, 38]. Recent studies suggest that the sputum surface properties may be more important determinants of cough clearability than viscoelasticity [7,39-41]. The presence of a bilayer at the gel-sol interface may also be an important determinant of mucociliary clearance, by facilitating the sliding of the gel phase over the sol phase [10, 12]. It is possible that the administration of exogenous surfactant enhances the formation of bilayers between mucus and periciliary fluid, and may also add to the number of reticulated surfactant structures in the periciliary fluid.

Thus, in addition to the notion that a surfactant bilayer between the two airway phases (gel and sol) may facilitate sliding of the gel phase on the sol phase, adding surfactant structures may change fluid dynamic properties, including dynamic viscosity of the periciliary fluid, enhancing the transfer of kinetic energy between the cilia and the mucous layer. Unfortunately, at present, very little is known about the mechanisms involved in the formation of bilayer structures in the periciliary fluid. If the mucus does not exist in a compact blanket, but takes the form of discrete aggregates of droplets or flakes, as has been suggested by VAN As [42], we may speculate that bilayers are formed from the monolayers at the airmucus interface and from monolayers extended directly over the periciliary fluid at gaps between mucous flakes. Bilayers could then be formed by a combination of translational and rotational motion of mucous flakes.

In summary, our findings clearly demonstrate that, in addition to the vital role that surfactants play in the periphery of the lung, surfactants also appear to be of great importance in airway mucociliary clearance. The stimulation of TMV after surfactant treatment may be due to the improvement of mucus surface properties, leading to an enhancement of ciliary function, either due to a decrease in adhesiveness between mucus and cilia or perhaps to alterations in periciliary fluid viscosity. There are potential implications of these findings in the clinical setting that could provide a new therapeutic approach towards improving impaired mucociliary clearance in such diseases as cystic fibrosis or chronic bronchitis, in which the common element is airway mucus clearance dysfunction.

\footnotetext{
Acknowledgements: The authors would like to thank W. Boyd of the University of Alberta and O. Ramirez and T. Dian of St. Louis University for their technical assistance. T. Curstedt (Karolinska Institute, Stockholm, Sweden) provided the sample of Curosurf.
}

\section{References}

1. Lucas AM, Douglas LC. Principles underlying ciliary activity in the respiratory tract. Arch Otolaryngol 1934; 20: $528-541$.

2. Widdicombe JG. Airway mucus. Eur Respir J 1989; 2: $107-115$

3. King M. Mucus, mucociliary clearance and coughing. In: Bates DV, ed. Respiratory Function in Disease. 3rd Edn. Philadelphia, Saunders, 1989; pp. 69-78.

4. King M. Viscoelastic properties of airway mucus. Fed Proc 1980; 39: 3080-3085.

5. Allegra L, Bossi R, Braga P. Influence of surfactant on mucociliary transport. Eur J Respir Dis 1985; 67 (Suppl. 142): 71-76.

6. Morgenroth K, Bolz J. Morphological features of the interaction between mucus and surfactant on the bronchial mucosa. Respiration 1985; 47: 225-231.

7. Girod S, Galabert C, Pierrot D, et al. Role of phospholipid lining on respiratory mucus clearance by cough. $J$ Appl Physiol 1991; 71: 2262-2266.

8. Rubin BK, Ramirez O, King M. The role of mucus rheology and transport in neonatal respiratory distress syndrome and the effect of surfactant therapy. Chest 1992; 101: 1080-1085.

9. Rubin BK. A superficial view of mucus and the cystic fibrosis defect. Pediatr Pulmonol 1992; 13: 4-5. 
10. Gehr P, Schürch S, Berthiaume Y, Im Hof V, Geiser M. Particle retention in airways by surfactant. J Aerosol Med 1990; 3, 27-43.

11. Mercer RR, Russell ML, Crapo JD. Mucous lining layers in human and rat airways. Am Rev Respir Dis 1992; 145: A355.

12. Schürch S, Gehr P, Im Hof V, Geiser M, Green FHY. Surfactant displaces particles toward the epithelium in airways and alveoli. Respir Physiol 1990; 80: 17-32.

13. Auten RL, Watkins RH, Shapiro DL, Horowitz S. Surfactant apoprotein A (SP-A) is synthetized in airway cells. Am J Respir Cell Mol Biol 1990; 3: 491-496.

14. Girod S, Fuchey C, Galabert C, et al. Identification of phospholipids in secretory granules of human submucosal gland respiratory cells. J Histochem Cytochem 1991; 39: 193-198.

15. Merrit TA, Hallman M. Surfactant replacement: a new era with many challenges for neonatal medicine. Am J Dis Child 1988; 142: 1333-1339.

16. Phibbs RH, Ballard RA, Clements JA, et al. Clinical trial of synthetic surfactant Exosurf for prophylaxis and early treatment of hyaline membrane disease. Pediatrics 1991; 88: 1-9.

17. Robertson B, Curstedt T, Johansson J, Jörnvall H, Kobayashi T. Structural and functional characterization of porcine surfactant isolated by liquid-gel chromatography. Prog Respir Res 1990; 25: 237-246.

18. King M, Kelly S, Cosio M. Alteration of airway reactivity by mucus. Respir Physiol 1985; 62: 47-59.

19. King M. Magnetic microrheometer. In: Braga PC, Allegra L, eds. Methods in Bronchial Mucology. New York, Raven Press, 1988, pp. 73-83.

20. Tomkiewicz RP, App EM, Coffiner M, Fossion J, Maes $\mathrm{P}$, King M. Mucolytic treatment with N-acetylcysteine L-lysinate metered-dose inhaler in dogs: airway epithelial function changes. Eur Respir J 1994; 7: 8187.

21. Holma B, Tomkiewicz RP, King M. Cilia beat frequency and mucociliary velocity in live frogs by a videoscopic method: effect of $\mathrm{N}$-acetylcysteine and its lysine derivative. Am Rev Respir Dis 1992; 145: A800.

22. Rensch H, von Seefeld H, Gebhardt KF, Renzow D, Sell PJ. Stop and go particle transport in the peripheral airways. Respiration 1983; 44: 346-350.

23. Lachmann B. Possible function of bronchial surfactant Eur J Respir Dis 1985; 67 (Suppl. 142): 49-60.

24. Geiser M, Im Hof V, Gehr P, Cruz-Orive LM. Histological and stereological analysis of particle retention in the conducting airways of hamster lungs. J Aerosol Med 1990; 3: 131-145.

25. Holm BA, Enhorning G, Notter RH. A mechanism by which plasma proteins inhibit surfactant function. Chem Phys Lipids 1988; 49: 49-55.

26. Schürch S, Schürch D, Curstedt T, Robertson B. Surface activity of lipid extract surfactant in relation to film area compression and collapse. J Appl Physiol 1994; (in press).
27. Im Hof V, Schürch S, Straub R, Gehr P. Surfactant in the trachea of the horse. Eur Respir J 1990; 3: 257s.

28. Espinosa EF, Shapiro AH, Fredberg JJ, Kamm RD. Spreading of exogenous surfactant in an airway. $J$ Appl Physiol 1993; 75: 2028-2039.

29. Schürch S, Bachofen $\mathrm{H}$. Biophysical aspects in the design of a therapeutic surfactant. In: Robertson B, Taeusch HW, eds. Surfactant Therapy for Lung Disease. New York, Marcel Dekker, 1994; (in press).

30. Kakuta Y, Sasaki H, Takishima T. Effect of artificial surfactant on ciliary beat frequency in guinea-pig trachea. Respir Physiol 1991; 83: 313-322.

31. Girod de Bentzmann S, Pierrot D, et al. Distearoyl phosphatidylglycerol liposomes improve surface and transport properties of CF mucus. Eur Respir J 1993; 6: 1156-1161.

32. Motta C, Touillon AM, Simon G, Dastugue B. Active lipid: a potential fluidifier of cystic fibrosis mucus. In: Lawson D, ed. Cystic Fibrosis: Horizons. Chichester, Wiley, 1984; p. 337.

33. Galabert C, Jacquot J, Zahm JM, Puchelle E. Relationship between lipid content and the rheological properties of airway secretions in cystic fibrosis. Clin Chim Acta 1987; 164: 139-149.

34. De Sanctis GT, Rubin BK, Ramirez O, King M. Ferret tracheal mucus rheology, clearability and volume following administration of substance $\mathrm{P}$ or methacholine. Eur Respir J 1993; 6: 76-82.

35. King M, Engel LA, Macklem PT. Effect of pentobarbital anesthesia on rheology and transport of canine tracheal mucus. J Appl Physiol : Respirat Environ Exercise Physiol 1979; 46: 504-510.

36. Rubin BK, Finegan B, Ramirez O, King M. General anesthesia does not alter the viscoelastic or transport properties of human respiratory mucus. Chest 1990; 98: 101-104.

37. Olivier K, Bennett W, Hohneker K, Zeman K, Boucher $\mathrm{R}$, Knowles M. Acute safety and effects on mucociliary clearance of uridine 5'-triphosphate (UTP) \pm amiloride in humans. Pediatr Pulmonol 1994: (in press).

38. Girod S, Zahm JM, Plotkowski C, Beck G, Puchelle E. Role of the physicochemical properties of mucus in the protection of the respiratory epithelium. Eur Respir $J$ 1992; 5: 477-487.

39. King M, Zahm JM, Pierrot D, Vaquez-Girod S, Puchelle E. The role of mucus gel viscosity, spinnability, and adhesive properties in clearance by simulated cough. Biorheology 1989; 26: 737-745.

40. Zahm JM, Pierrot D, Vaquez-Girod S, Duvivier C, King $\mathrm{M}$, Puchelle E. The role of mucus sol phase in clearance by simulated cough. Biorheology 1989; 26: 747752.

41. Agarwal M, King M, Rubin BK, Shukla JB. Mucus transport in a miniaturized simulated cough machine: effect of constriction and serous layer simulant. Biorheology 1989; 26: 977-988.

42. Van As A. Pulmonary clearance mechanisms: a reappraisal. Am Rev Respir Dis 1977; 115: 721-726. 